\title{
Compressed Sensing-Based Order Analysis for Blade Tip Timing Signals Measured at Varying Rotational Speed
}

\author{
Suiyu Chen $\left(\mathbb{D}\right.$, Yongmin Yang $\left(\mathbb{D}\right.$, Haifeng Hu $\left(\mathbb{D}\right.$, Fengjiao Guan $\mathbb{D}^{D}$, Guoji Shen, \\ and Faming Yang \\ Laboratory of Science and Technology on Integrated Logistics Support, National University of Defense Technology, \\ Changsha 410073, China \\ Correspondence should be addressed to Yongmin Yang; yangyongmin@163.com
}

Received 30 October 2019; Revised 7 September 2020; Accepted 31 October 2020; Published 26 November 2020

Academic Editor: Fabio Rizzo

Copyright (c) 2020 Suiyu Chen et al. This is an open access article distributed under the Creative Commons Attribution License, which permits unrestricted use, distribution, and reproduction in any medium, provided the original work is properly cited.

\begin{abstract}
Monitoring the vibrations of high-speed rotating blades is significant to the security of turbomachineries. Blade tip timing (BTT) is considered as a promising technique for detecting blade vibrations without contact online. However, extracting blade vibration characteristics accurately from undersampled BTT signals measured at varying rotational speed (VRS) has become a big challenge. The existing two methods for this issue are restricted within the order bandwidth limitation and require prior information and precise sensor installation angles, which is often unpractical. To overcome these difficulties, a compressed sensing-based order analysis (CSOA) method was proposed. Its feasibility comes from the sparsity of BTT vibration signals in the order domain. The mathematical model for the proposed method was built, and the optimizing principles for sensor number and sensor arrangement were given. Simulated and experimental results verified the feasibility and advantages of the proposed method that it could extract order spectrum accurately from BTT vibration signals measured at VRS without the drawbacks in the existing two methods.
\end{abstract}

\section{Introduction}

High-speed rotating blades are critical components in turbomachinery, such as gas turbine engines and aircraft engines [1]. They often suffer from multifrequency vibrations resulted from various exciting forces, including centrifugal force, aerodynamic force, and impact force. These vibrations reduce the service life of the blades and may lead to cracks and even fractures, resulting in catastrophic accidents [2]. Thus, it is quite necessary to monitor blade conditions online to find the damages in time. The traditional approach is to install strain gauges on the blades, which is rather costly because it requires high-quality telemetry units or slip rings and complex installation process $[3,4]$.

BTT technique is an alternative to strain gauges and has become a hot issue due to its advantages of noncontact measurement, easy installation, and the ability to monitor all the blades simultaneously [5]. The BTT system precisely measures the time of arrival (TOA) of each blade tip at each sensor which is installed in the casing around the bladed disk [6]. A reference sensor is mounted near the spindle to provide a reference time once per revolution, from which the TOA of nonvibrating blade can be derived [7]. The vibration displacement can be obtained via the difference in the TOAs of the vibrating blade and nonvibrating blade through which blade parameters including vibrating frequency and mode can be analyzed [8]. However, the BTT vibration signal is undersampled because the overall sampling frequency, which is determined by rotational speed and limited sensor number, is usually less than twice the maximum blade vibrating frequency [9-11].

Until now, some methods have been proposed to extract blade vibration characteristics from undersampled BTT signals. Witos and Wisnioch [12] in the Polish Air Force have been studying the BTT technique since 1993 and conducted excellent researches. They extracted TOA signal components and diagnostic symptoms by a numerical process. Moreover, they employed a phase mapping method for analysis. Beauseroy and Lengellé [13] proposed a new analytical method for multifrequency BTT signals with a group of regularly spaced optical sensors. Salhi et al. [14] 
introduced a subspace modelling method to identify the natural frequency of blades using two sensors installed at the interval of $180^{\circ}$. Lin et al. [15] presented a reconstruction algorithm for multimode blade vibration signals based on the sparse representation theorem. Hu et al. [9] reconstructed BTT signals measured by nonuniformly placed sensors by considering it as a bandpass signal. Pan et al. [16] proposed a method to extract the spectrum of multifrequency blade vibrations and alleviate the measurement uncertainties of BTT signals. These methods are all based on time domain and constant speed assumption. However, the rotational speed may change frequently and result in inconstant sampling frequency, which invalidates these methods. Besides, the methods in [13-16] require strictly precise sensor installation angles, which are difficult to realize.

Although extracting blade vibration characteristics from BTT signals measured at VRS has become an obstacle, few methods were proposed for solving this problem. Zhan et al. [17] proposed an interpolation algorithm to reconstruct blade vibration signals measured by uniformly arranged sensors, which made compensation for the effects of VRS. However, this method required strictly precise sensor installation angles and prior information, including central frequency and signal bandwidth. Chen et al. [18] improved the work in [17] and proposed two-order analysis-based reconstruction methods for BTT vibration signals at VRS by considering the signals as bandpass. Compared to [17], the first method in [18] substituted angular domain for time domain and still required uniformly installed sensors. The second method accurately captured high-engine orders of blade vibrations by using two sensors with arbitrary installation angles. However, neither of them can reconstruct vibration signals without the prior information of the central engine order and order bandwidth of the signal. Besides, the order bandwidth is quite limited by a sensor number.

Compressed sensing (CS) theory was proposed to accurately reconstruct spectrum-blind (no prior information) sparse signals from undersampled samples [19-22]. Since the BTT signals measured at VRS have sparsity in the order domain, this paper proposed an order analysis method to obtain the order spectrum of BTT vibration signals at VRS based on CS theory. Compared to the existing methods for BTT vibration signals at VRS in $[17,18]$, the proposed method has three major advantages: firstly, it requires no prior information of the central engine order and order bandwidth of signals; secondly, it is still feasible to the signals exceeding the order bandwidth limitation as stated in [18]; and thirdly, it allows considerable installation angle errors.

This paper is organized as follows: the BTT mechanism was elaborated in Section 2. A CSOA mathematical model was built in Section 3. Then, the optimizing principles for the number and arrangement of the sensors were provided in Section 4 . The existing methods in [18] were described in detail in Section 5. Simulations in Section 6 and experiments in Section 7 were conducted to verify the feasibility of the proposed method and the advantages over the existing methods. Finally, conclusions were drawn in Section 8.

\section{BTT Mechanism}

As shown in Figure 1, several sensors are mounted in the stationary casing around a rotating bladed disk to measure the TOAs of each blade at each sensor. Sensor $r$ is installed close to the spindle with a reflective tape to provide a reference time for each revolution. The time pulse signals are recorded for the first time when the reflective tape passes Sensor $r$. The TOAs and the reference time are usually acquired by extracting the rising edge time or falling edge time from the recorded time pulse signals.

Considering Sensor $r$ as the reference position, the angular positions of the $i$ th sensor (Sensor $i$ ) and the $b$ th blade (Blade $b$ ) at the time when the reflective tape passes Sensor $r$ are represented as $\alpha_{i}$ and $\theta_{b}$, respectively. At the $n$th revolution, the actual TOA of Blade $b$ at Sensor $i$ and the duration of rotation are denoted as $t_{i, b}[n]$ and $T[n]$, respectively. If the blades do not vibrate, the TOA of Blade $b$ at Sensor $i$ during the $n$th revolution is termed as the expected TOA, $\tilde{t}_{i, b}[n]$, which can be derived by

$$
\tilde{t}_{i, b}[n]=\frac{\alpha_{i}-\theta_{b}}{2 \pi f_{r}[n]}+\sum_{k=0}^{n-1} T[k], \quad T[0]=0,
$$

where $f_{r}[n]$ is the average rotational frequency from the time the $n$th revolution starts to the expected TOA, which can be derived by the spline interpolation method.

Practically, the blades usually vibrate under various incentives, causing a deflection when arriving at the sensors, which results in a time difference between the actual TOA and expected TOA. The deflection of Blade $b$ when it arrives at Sensor $i$ during the $n$th revolution can be obtained by

$$
y_{i}[n]=2 \pi f_{r}[n] R_{b}\left(\tilde{t}_{i, b}[n]-t_{i, b}[n]\right),
$$

where $R_{b}$ denotes the distance from the axis to the tip of Blade $b$. Thus, by matching the sequences $\left\{\tilde{t}_{i, b}[n]\right\}$ and $\left\{y_{i}[n]\right\}$, a time-based vibrating displacement sequence of Blade $b$ arriving at Sensor $i$ can be acquired. Through analyzing all the sequences, the vibrating characteristics of blades can be obtained, thus providing information for detecting blade damages in real time.

\section{CSOA Method}

Consider a real continuous angular-domain blade vibration signal $x(\theta)$ for Blade $b$, and $m$ sensors are installed in the casing. Here, the expected rotating angles (no blade vibrations) at which the blades pass the sensors are considered as angular sampling points. Since the installation angles remain unchanged during the measurement process, the sampling intervals of a single sensor on the angular domain are identical. A sampling vibrating signal of Blade $b$ measured by Sensor $i(1 \leq i \leq m)$ can be described as follows: 


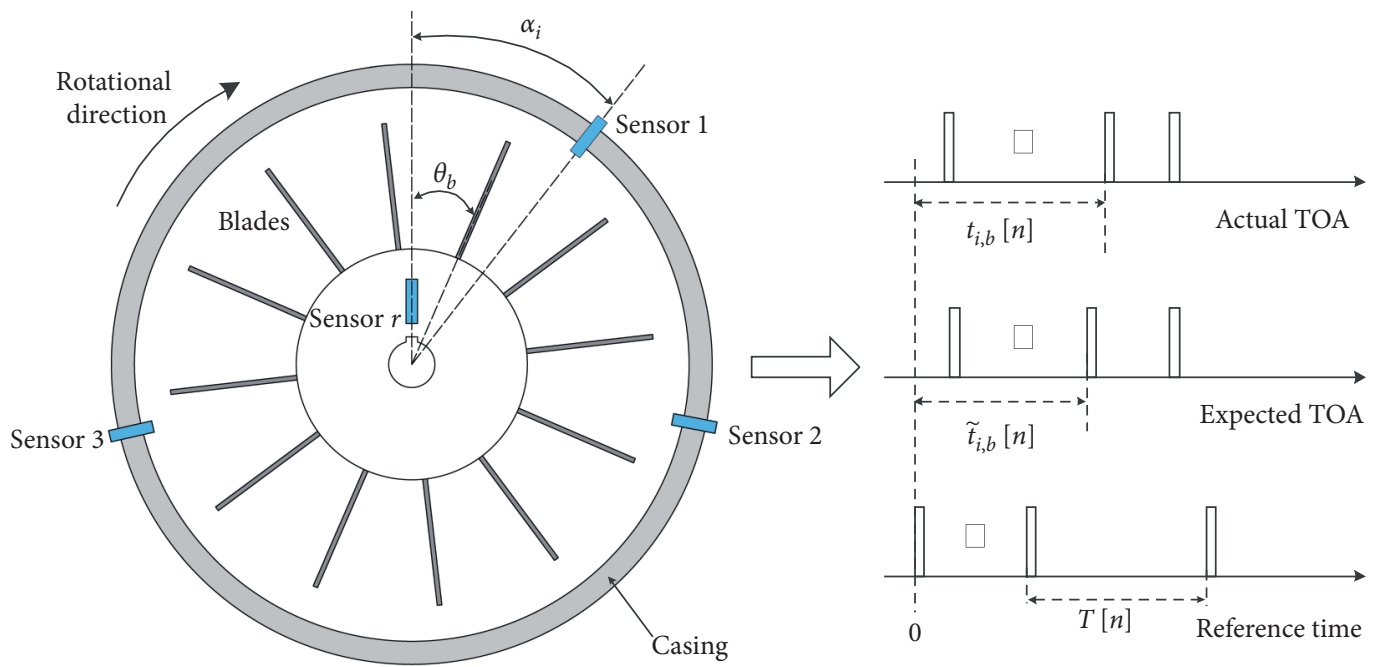

Figure 1: Schematic of BTT mechanism.

$$
\begin{array}{cl}
y_{i}[n]=\sum_{\theta=-\infty}^{\infty} & x(\theta) \delta\left(\theta-2 \pi(n-1)-\left(\alpha_{i}-\theta_{b}\right)\right), \\
& n=1,2, \ldots
\end{array}
$$

Let $\beta_{i, b}=\alpha_{i}-\theta_{b}$, then equation (3) can be rewritten as

$$
y_{i}[n]=\sum_{\theta=-\infty}^{\infty} x(\theta) \delta\left(\theta-2 \pi(n-1)-\beta_{i, b}\right) .
$$

The sampling function can be expressed as

$$
p_{i}(\theta)=\sum_{n=-\infty}^{\infty} \delta\left(\theta-2 \pi(n-1)-\beta_{i, b}\right) .
$$

Since $p_{i}(\theta)$ is a periodic function with the sampling interval, $T_{s}=2 \pi$, it has a Fourier expansion:

$$
p_{i}(\theta)=\sum_{l=-\infty}^{\infty} c_{i, l} e^{j l \theta},
$$

where $c_{i, l}$ is the Fourier coefficient, which can be given by

$$
c_{i, l}=\frac{1}{T_{s}} \int_{0}^{T_{s}} p_{i}(\theta) e^{-j\left(2 \pi / T_{s}\right) l \theta} \mathrm{d} \theta=\frac{1}{2 \pi} e^{-j l \beta_{i, b}} .
$$

Let $x_{i}(\theta)=x(\theta) p_{i}(\theta)$. Its Fourier transform can be calculated as

$$
\begin{aligned}
X_{i}(E) & =\int_{-\infty}^{\infty} x_{i}(\theta) e^{-j E \theta} \mathrm{d} \theta=\int_{-\infty}^{\infty} x(\theta)\left(\sum_{l=-\infty}^{\infty} c_{i, l} l^{j l \theta}\right) e^{-j E \theta} \mathrm{d} \theta \\
& =\sum_{l=-\infty}^{\infty} c_{i, l} \int_{-\infty}^{\infty} x(\theta) e^{-j(E-l) \theta} \mathrm{d} \theta=\sum_{l=-\infty}^{\infty} c_{i, l} X(E-l),
\end{aligned}
$$

where $E$ is the engine order. The discrete Fourier transform (DFT) of $y_{i}[n]$ is given by

$$
Y_{i}(E)=\sum_{n=-\infty}^{\infty} y_{i}[n] e^{-j 2 \pi E n} .
$$

Equation (9) can be tied to equation (8) since they are transforms of the identical signal, which can be formulated as

$$
Y_{i}(E)=\sum_{l=-L_{0}}^{L_{0}} c_{i, l} X(E-l), \quad E \in\left[\frac{-1}{2}, \frac{1}{2}\right],
$$

where $E \in[-1 / 2, t 1 / 2]$ is set to avoid the aliasing effect according to Shannon's sampling theorem since the vibrating displacement of Blade $b$ is sampled once per revolution by Sensor i. $L_{0}$ is an integer chosen to cover all the nonzero terms in order spectrum, which can be obtained by

$$
\begin{aligned}
-\frac{1}{2}+\left(L_{0}+1\right) & \geq E_{\max } \\
\Rightarrow L_{0} & \geq E_{\max }-\frac{1}{2},
\end{aligned}
$$

where $E_{\max }$ is the maximum order of the signal. It can be seen from equation (10) that $Y_{i}(E)$ can be considered as a linear combination of shifted copies of $X(E)$. Thus, the CSOA mathematical model can be derived as follows by rewriting equation (10) in matrix form:

$$
\mathbf{Y}(E)=\mathbf{\Phi} \mathbf{z}(E), \quad E \in\left[\frac{-1}{2}, \frac{1}{2}\right],
$$

where

$$
\begin{aligned}
\mathbf{Y}(E) & =\left[Y_{1}(E), Y_{2}(E), \ldots, Y_{m}(E)\right]^{T}, \\
\mathbf{z}(E) & =\left[z_{1}(E), z_{2}(E), \ldots, z_{L}(E)\right]^{T}, \\
L & =2 L_{0}+1,
\end{aligned}
$$




$$
z_{k}(E)=X\left(E+k-L_{0}-1\right), \quad 1 \leq k \leq L
$$

The $m \times L$ sensing matrix $\Phi$ is constituted by $\Phi\left(i, L_{0}+l+1\right)=c_{i,-l}=\frac{1}{2 \pi} e^{j l \beta_{i, b}}, \quad 1 \leq i \leq m,-L_{0} \leq l \leq L_{0}$.

Since the BTT signal is sparse in order domain, the analysis of the signal can be transformed into a typical CS problem as follows:

$$
\begin{aligned}
P 0: \mathbf{z}_{\text {opt }}(E)= & \underset{\mathbf{z}(E)}{\arg \min }\|\mathbf{z}(E)\|_{0} \text { s.t. } \mathbf{Y}(E)=\mathbf{\Phi z}(E), \\
& E \in\left[\frac{-1}{2}, \frac{1}{2}\right] .
\end{aligned}
$$

Figure 2 provides a schematic of the proposed CS mathematical model. The abscissa and the ordinate of the graph, respectively, denote $E+k-L_{0}-1$ and $z_{k}(E)$ with varying $k . L_{0}$ is set to cover the order scale of the blade vibration signal $\left[-E_{\max }, E_{\max }\right]$. It is considered the blade vibration signal has several orders $\left(E_{1}, E_{2}, \ldots, E_{\max }\right)$ arbitrarily distributed in the order domain. It can be seen that the order spectrum of the blade vibration signal can be extracted by finding the nonzero terms in the order scale, which can be transformed into the typical CS problem shown in equation (18) and solved by existing CS algorithms.

Since the CS algorithms can find all the nonzero terms in ignorance of their distribution, the proposed method requires no prior information of central frequency and order bandwidth of the blade vibration signal. In addition, the key to solve this CS problem is constructing the sensing matrix $\Phi$, which comes down to the choices of $m, L$, and $\alpha_{i}(1 \leq i \leq m)$.

\section{Parameter Optimization}

It can be concluded from Section 3 that the parameter optimization for the sensing matrix contributes greatly to successfully extract the order spectrum from the blade vibration signal. $L$ can be chosen via equation (15), while the optimizations of $m$ and $\alpha_{i}$ require further investigations. This is an optimizing problem of the number and arrangement of the sensors.

4.1. Sensor Number Optimization. According to the CS theory $[19,20], \mathbf{z}(E)$ is the unique sparsest solution of equation (18) and can be accurately extracted with a great possibility if it satisfies in the following equation:

$$
\|\mathbf{z}(E)\|_{0}<\frac{\operatorname{spark}(\boldsymbol{\Phi})}{2}
$$

where spark $(\Phi)$ is the smallest number of columns from $\Phi$ which are linearly dependent [23-25]. According to this definition, $2 \leq \operatorname{spark}(\Phi) \leq m+1$. Thus, the sensor number can be related to $\|\mathbf{z}(E)\|_{0}$ as follows:

$$
\|\mathbf{z}(E)\|_{0}<\frac{m+1}{2}
$$

However, equation (20) is a sufficient but not necessary condition for the unique sparsest solution of equation (18) and great possibility of recovering $\mathbf{z}(E)$. Thus, sensor numbers which are smaller than $2\|\mathbf{z}(E)\|_{0}-1$ may also be allowable.

4.2. Sensor Arrangement Optimization. According to $[26,27], \mathbf{z}(E)$ can be accurately recovered by orthogonal matching pursuit (OMP) $[28,29]$ or basis pursuit (BP) $[30,31]$ algorithms if it satisfies the following condition [25]:

$$
\|\mathbf{z}(E)\|_{0}<\frac{1}{2}\left(1+\frac{1}{\mu(\Phi)}\right)
$$

where $\mu(\Phi)$ is the two-sided coherence [32] of $\Phi$, which is given by

$$
\mu(\Phi)=\max _{1 \leq i, j \leq L, i \neq j} \frac{\left|\boldsymbol{\varphi}_{i}^{T} \boldsymbol{\varphi}_{j}\right|}{\left\|\varphi_{i}\right\|_{2} \cdot\left\|\varphi_{j}\right\|_{2}}
$$

where $\varphi_{i}$ is the $i$ th column vector of $\Phi$. It can be inferred from equation (21) that the smaller the $\mu(\Phi)$ is, the larger the $\|\mathbf{z}(E)\|_{0}$ is allowed, which in fact allows a greater number of the blade vibration engine orders. Thus, the sensor arrangement principle is to choose proper installation angles to minimize $\mu(\Phi)$ as much as possible.

\section{Detailed Description of the Existing Method}

The existing two methods presented by Chen [18] considered the blade vibration signals as bandpass based on Shannon's sampling theorem in the angular domain. The first method uses several uniformly mounted sensors to obtain uniformly sampling signals in the angular domain with a constant sampling interval $2 \pi / m$. The first method can be expressed as

$$
\hat{s}(\theta)=\operatorname{Re}\left[\sum_{n=-\infty}^{\infty} \widetilde{s}[n] \sin c\left(E_{s} \theta-n\right) e^{j 2 \pi\left(E_{0} / E_{s}\right)\left(E_{s} \theta-n\right)}\right],
$$

where $\widehat{s}(\theta)$ is the reconstructed signal and $\widetilde{s}[n]$ is the Hilbert transform of the sampled blade vibration signal. $E_{0}$ is the central order, and $E_{s}=m$ denotes the sampling order. However, the order bandwidth of the signal cannot exceed $E_{s} / 2$ in equation (23). Besides, this method requires strictly precise installation angles to ensure uniform sampling in the angular domain.

The second method uses two nonuniformly mounted sensors. It is based on the second-order sampling theorem [33]. Its reconstruction formula is given by

$$
\widehat{\boldsymbol{s}}(\theta)=\sum_{n=-\infty}^{\infty}\left[s_{1}[n] h\left(\theta-\frac{n}{B}\right)+s_{2}[n] h\left(-\theta+\frac{n}{B}+\frac{\Delta \alpha}{2 \pi B}\right)\right],
$$




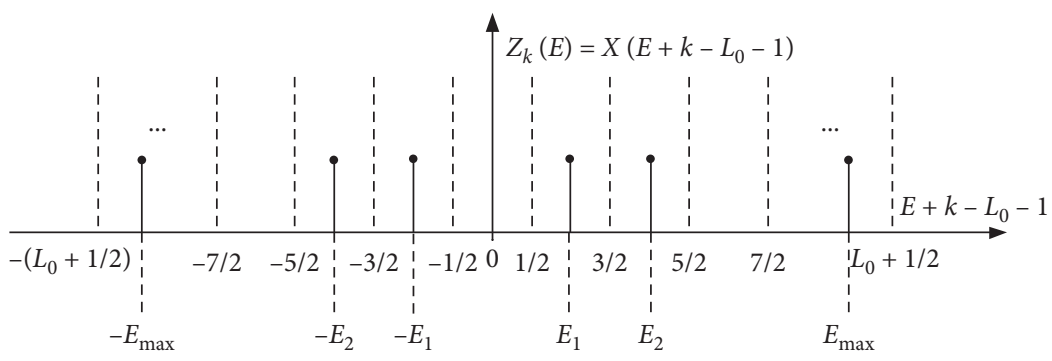

FIgURE 2: Schematic of the CSOA mathematical model.

where $s_{1}[n]$ and $s_{2}[n]$ are the blade vibration signals sampled by the two sensors, respectively. $\Delta \alpha$ is the angle between the two sensors. $B=1$ is the order bandwidth, and $h(\theta)$ can be given by

$$
\begin{aligned}
h(\theta)= & \frac{\cos \left[2 \pi\left(\lambda B-E_{\min }\right) \theta-\lambda \Delta \alpha / 2\right]-\cos \left(2 \pi E_{\min } \theta-\lambda \Delta \alpha / 2\right)}{2 \pi B \theta \sin (\lambda \Delta \alpha / 2)} \\
& +\frac{\cos \left[2 \pi\left(B+E_{\min }\right) \theta-(\lambda+1) \Delta \alpha / 2\right]-\cos \left[2 \pi\left(\lambda B-E_{\min }\right) \theta-(\lambda+1) \Delta \alpha / 2\right]}{2 \pi B \theta \sin [(\lambda+1) \Delta \alpha / 2]},
\end{aligned}
$$

where $\lambda=\left\lceil 2 E_{\min } / B\right\rceil$. The second method does not require precise installation angles. However, it cannot reconstruct blade vibration signal whose order bandwidth exceeds one.

\section{Numerical Simulations}

6.1. Simulated Blade Vibration Signal. It is assumed that each blade is identical and is not coupled with others. In addition, they vibrate in a single degree of freedom under the simulated synchronous exciting force, which can be given by

$$
F(t)=\sum_{i=1}^{3} F_{i} \cos \left(E_{i} \theta(t)+\varphi_{i}\right)
$$

where $F_{i}$ and $E_{i}$ are the amplitude and the order of the force, respectively. $\theta(t)$ is the rotating angle, which is calculated as follows:

$$
\theta(t)=2 \pi \int_{0}^{t} f_{r}(t) \mathrm{d} t
$$

where $f_{r}(t)$ is the rotational frequency varying with time.

A mathematical model for blade vibration can be derived as

$$
\ddot{x}=\frac{1}{m_{b}} \cdot[F(t)-c \dot{x}-k x],
$$

where $m_{b}, c$, and $k$ are the equivalent mass, damping coefficient, and elastic coefficient of the blade. $x$ is the blade tip displacement.

Based on equations (26)-(28), a simulated signal acquisition system for blade tip vibration at VRS was built, as shown in Figure 3. Chirps 1 3 generate swept frequency cosine with instantaneous frequencies equal to $E_{1}, E_{2}$, and $E_{3}$ times the rotational frequency, respectively. Chirp 4 provides swept frequency sine with an instantaneous frequency equal to the rotational frequency. The switches enable the system to output the blade tip displacements each time it satisfies the following condition:

$$
\theta(t)=2 \pi n+\alpha_{i}-\theta_{b}, \quad n=0,1, \ldots,
$$

where the angular position of the blade $\theta_{b}=0$ and the $i$ th sensor installation angle $\alpha_{i}$ is set in hit crossings. Port 1 outputs the original blade tip displacements, Port $2 \sim j$ outputs the displacements measured by the sensors, and Port $j+1$ is used for calculating the starting time sequences of each revolution.

6.2. Simulation for the Order Bandwidth Limitation. To verify that the CSOA method is still feasible to the BTT vibration signals which exceed the order bandwidth limitation of the existing methods in [18], simulated signals of different $E_{i}$ generated by the system in Figure 3 were analyzed. The simulation parameters are shown in Table 1 and have been optimized according to the theoretical analysis in Sections 3 and 4 . In addition, white Gaussian noise is added to the simulated signals.

The natural frequency of the blade can be calculated by

$$
f_{n}=\frac{1}{2 \pi} \sqrt{\frac{k}{m_{b}}}=636.6198 \mathrm{~Hz}
$$

Figure 4 shows the simulated blade tip displacements varying with the rotating frequency and the data sampled by the sensors. The blade vibrates resonantly when the excitation frequency reaches $f_{n} / E_{i}$.

Figure 5 shows the order spectrum obtained via the CSOA method. It is obvious that the order spectrums of the two sets of simulated blade tip displacements are accurately obtained. 


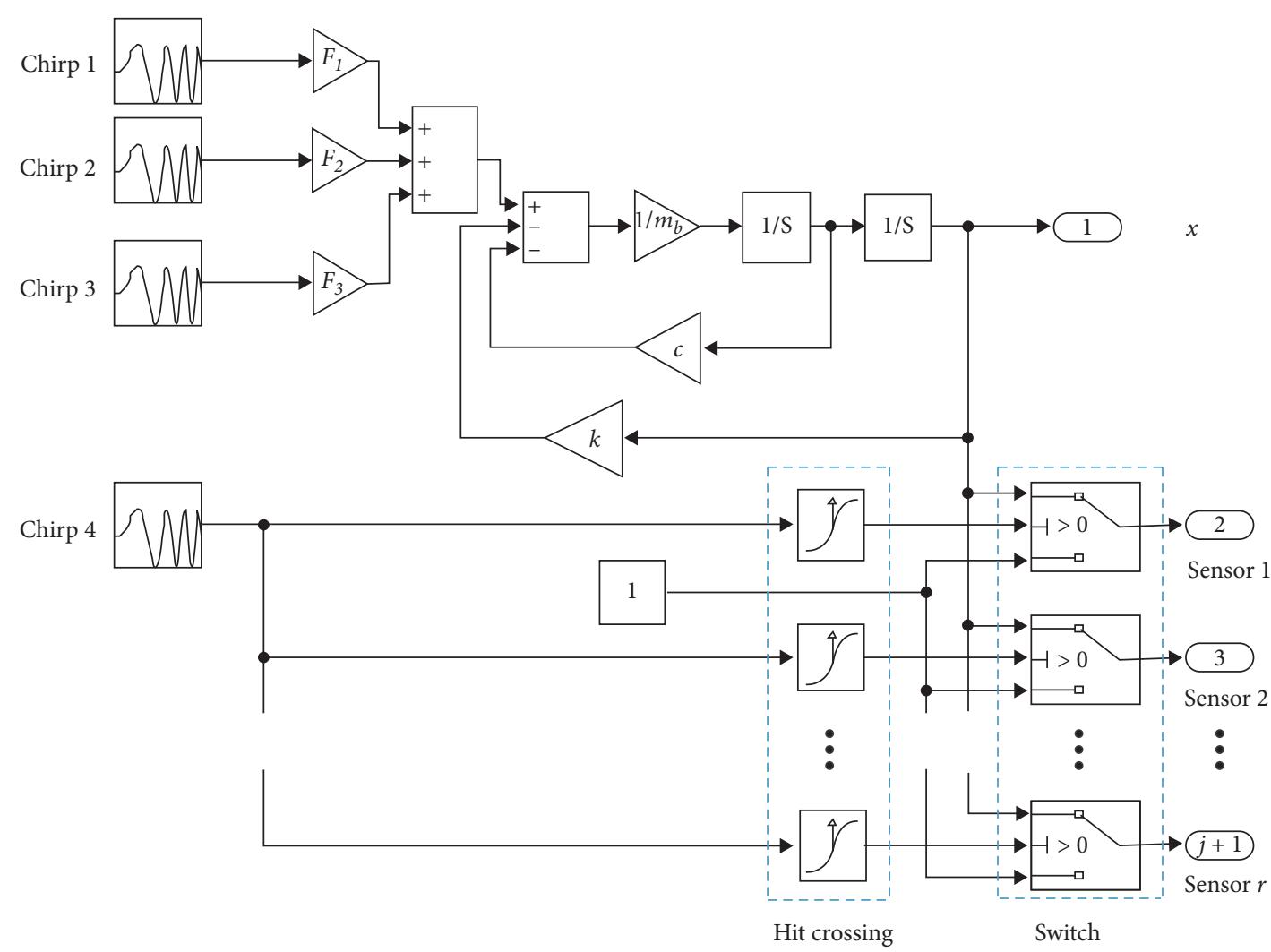

FIgURE 3: Simulated system for blade tip vibration at VRS.

TABLE 1: Simulation parameters.

\begin{tabular}{lc}
\hline Parameters & Values \\
\hline$\left\{E_{1}, E_{2}, E_{3}\right\}$ & $\{1,2,4\}$ and $\{1,2,13\}$ \\
$\left\{F_{1}, F_{2}, F_{3}\right\}$ & $\{6,8,10\} \mathrm{N}$ \\
$\left\{\varphi_{1}, \varphi_{2}, \varphi_{3}\right\}$ & $\{0,0,0\}$ \\
$m_{b}$ & $0.05 \mathrm{~kg}$ \\
$C$ & $10 \mathrm{~N} \cdot \mathrm{s} / \mathrm{m}$ \\
$K$ & $8 \times 10^{5} \mathrm{~N} / \mathrm{m}$ \\
Rotating frequency & $30 \sim 700 \mathrm{~Hz}$ \\
Frequency sweep & Linear \\
Time & $100 \mathrm{~s}$ \\
Sensor number & 7 \\
Installation angles & $\left\{32^{\circ}, 75^{\circ}, 105^{\circ}, 145^{\circ}, 197^{\circ}, 215^{\circ}, 324^{\circ}\right\}$ \\
SNR & 10 \\
\hline
\end{tabular}

For comparison, the data were also analyzed with the existing methods. The sensor number for the first method and $\Delta \alpha$ for the second method in [18] are set to 7 and $25^{\circ}$, respectively. The results are shown in Figure 6.

The simulation results suggested that the two methods in [18] failed to acquire the exact order spectrum of the signal beyond the bandwidth limitation. In conclusion, the CSOA method is effective whether the signal is within or exceeding the order bandwidth limitation of the existing methods proposed in [18]. Moreover, $L$ in CSOA can be enlarged to meet the needs of a larger order bandwidth.
6.3. Simulation for Installation Angle Errors. To demonstrate that the CSOA method allows considerable installation angle errors, the angles were set as $\alpha^{\prime}=\alpha+\Delta$, where $\Delta$ is the deviation from the expected installation angles $\alpha$ and randomly generated within $\left[-10^{\circ}, 10^{\circ}\right]$. Other parameters are shown in Table 1 . The simulation results via the CSOA method are shown in Figure 7.

In Figure 7, the order spectrum is precisely acquired despite the installation angle errors, which proves that the CSOA method is still effective under the condition of inaccurate installation angles. This characteristic is quite useful since the installation angle errors exist in practical applications.

\section{Experiments}

For further verification on the effects of the proposed CSOA method, an experimental rig was built, as shown in Figure 8. The rig consists of an electrical motor (installed under the test bench), a bladed disk with 32 uniformly distributed straight blades (labelled as Blades 1 32), a spindle, a casing, six uniformly distributed permanent magnets, and four optical fiber sensors (Sensors 1 3 were mounted on the casing in precise alignment along the circumstance, and Sensor $r$ was the reference sensor). The operating frequency of a high-speed data acquisition card is $5 \mathrm{MHz}$. Sensor $r$ was installed vertically, and the installation angles of Sensors 1 3 were set as $15^{\circ}, 143^{\circ}$, and $270^{\circ}$, respectively. These angles 

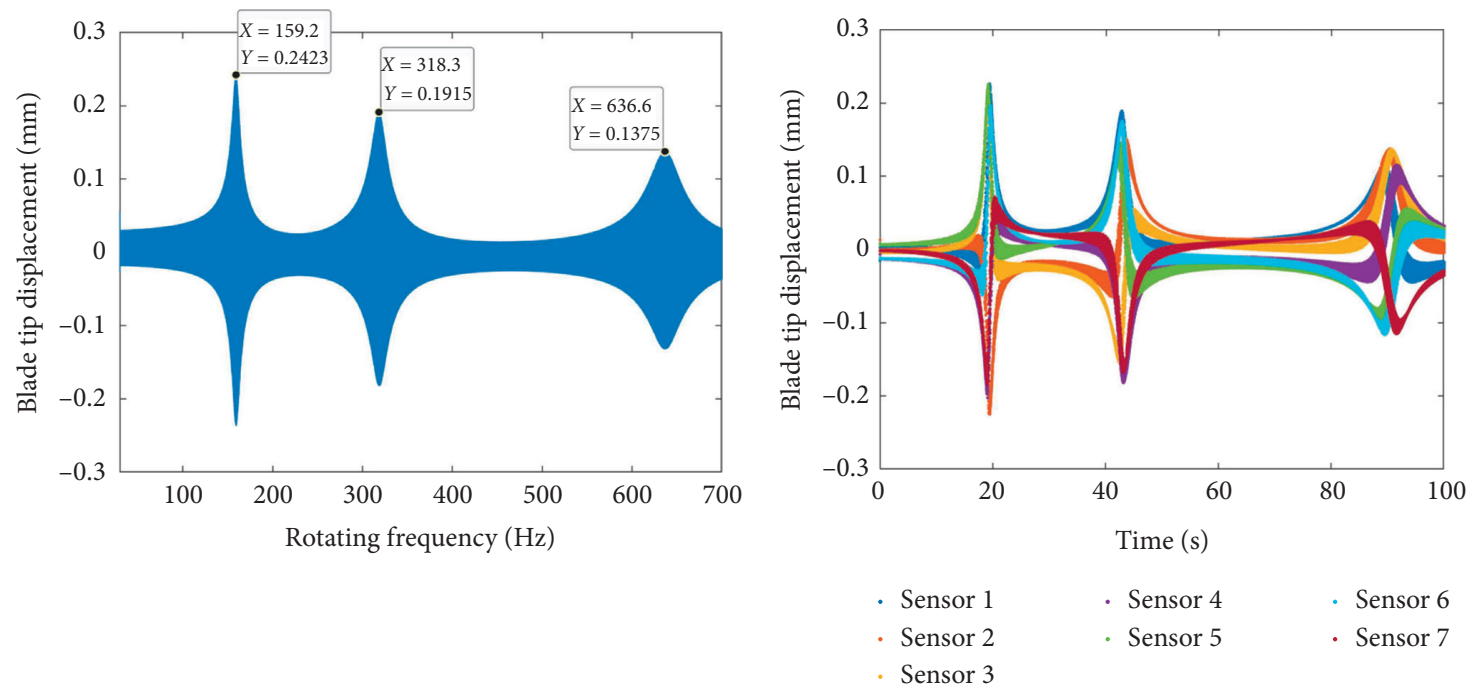

(a)
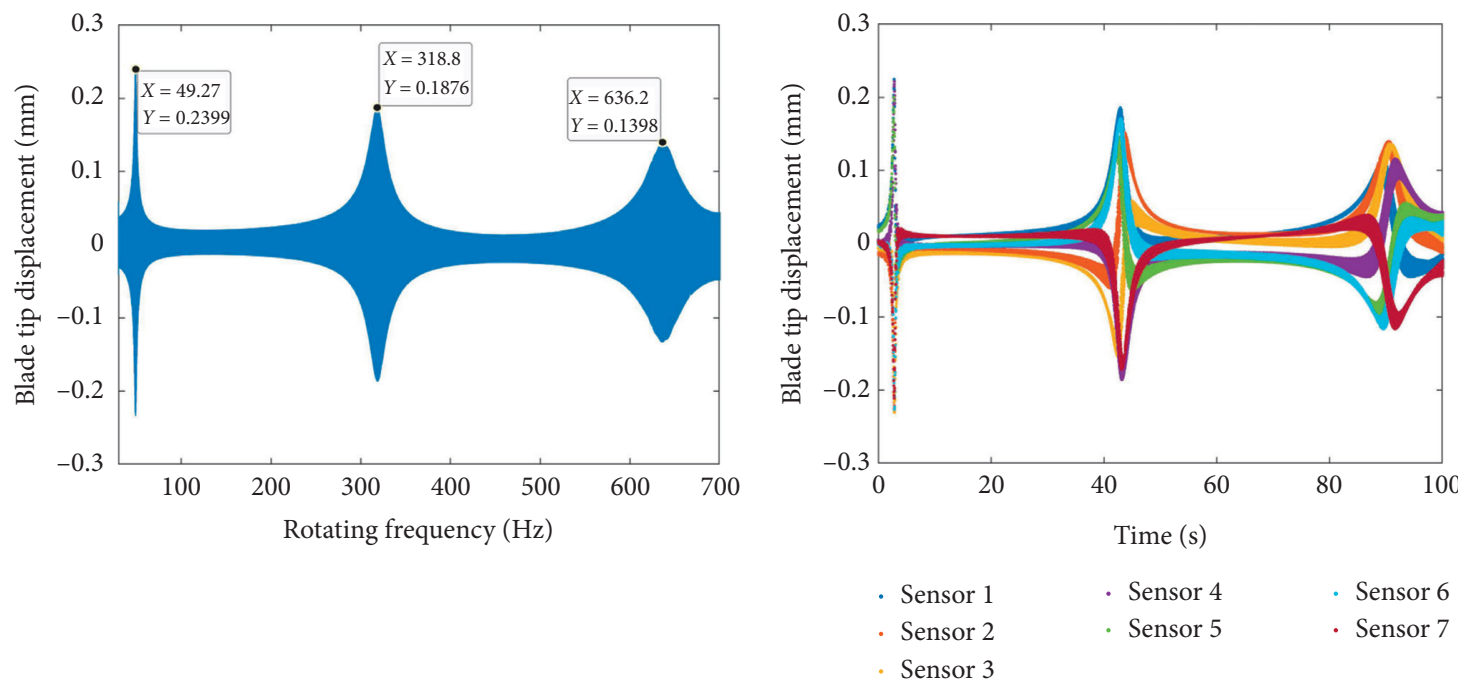

(c)

(d)

Figure 4: (a, c) Simulated blade tip displacement with $\left\{E_{1}=1, E_{2}=2, E_{3}=4\right\}$ and $\left\{E_{1}=1, E_{2}=2, E_{3}=13\right\}$, respectively; (b, d) data sampled by the sensors with $\left\{E_{1}=1, E_{2}=2, E_{3}=4\right\}$ and $\left\{E_{1}=1, E_{2}=2, E_{3}=13\right\}$, respectively.

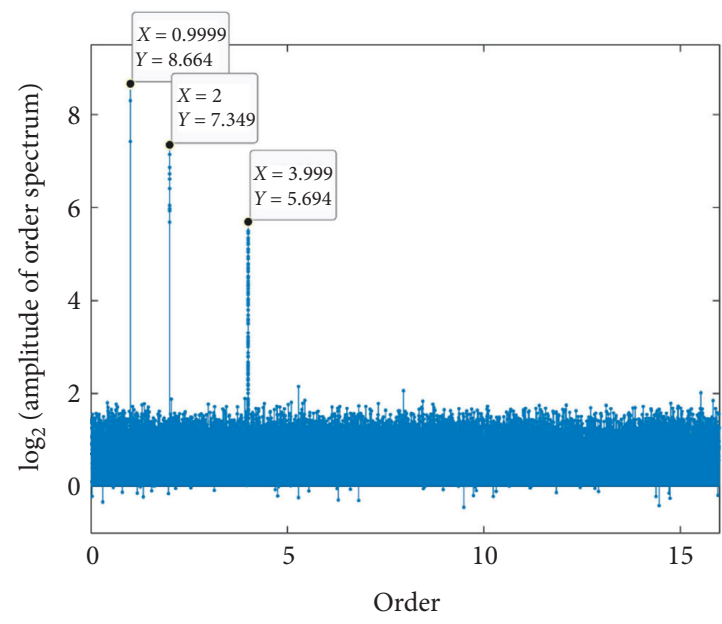

(a)

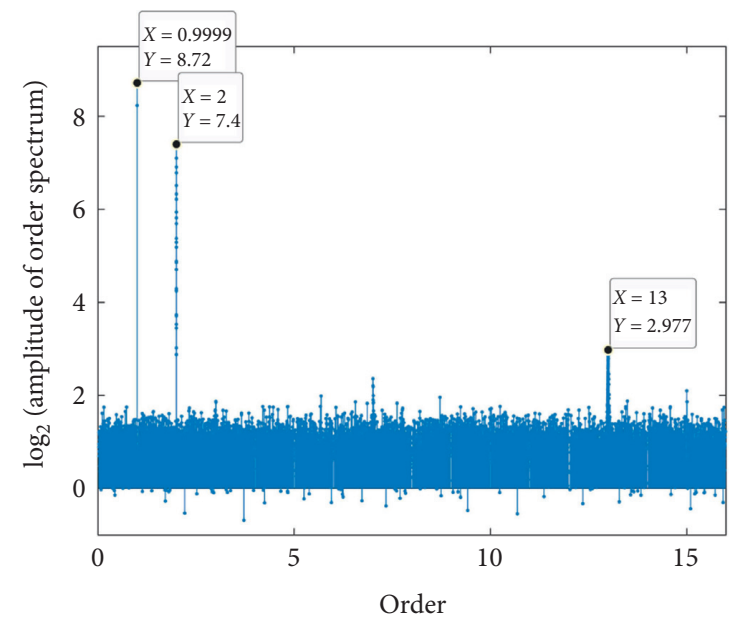

(b)

FIGURE 5: Order spectrums of the simulated blade tip displacements obtained via the CSOA method: (a) $\left\{E_{1}=1, E_{2}=2, E_{3}=4\right\}$; (b) $\left\{E_{1}=1, E_{2}=2, E_{3}=13\right\}$. 


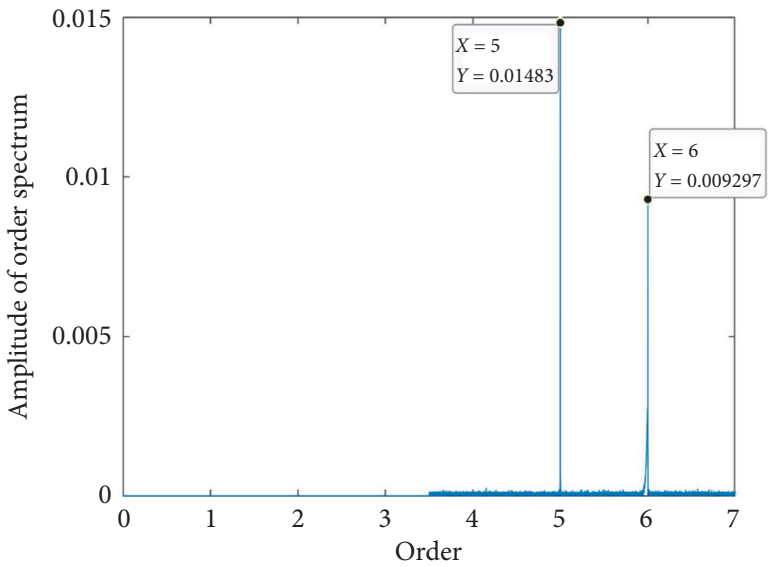

(a)

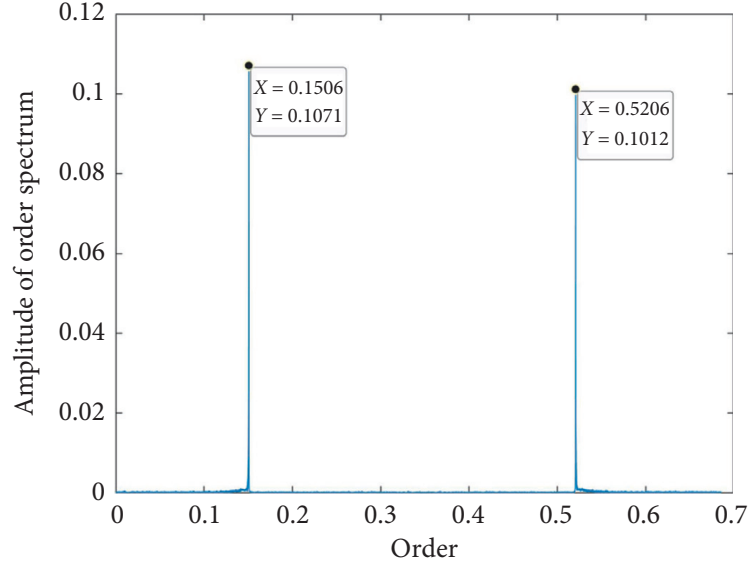

(b)

Figure 6: Order spectrum of the simulated blade tip displacements $\left\{E_{1}=1, E_{2}=2, E_{3}=13\right\}$ via (a) the first method and (b) the second method in [18].

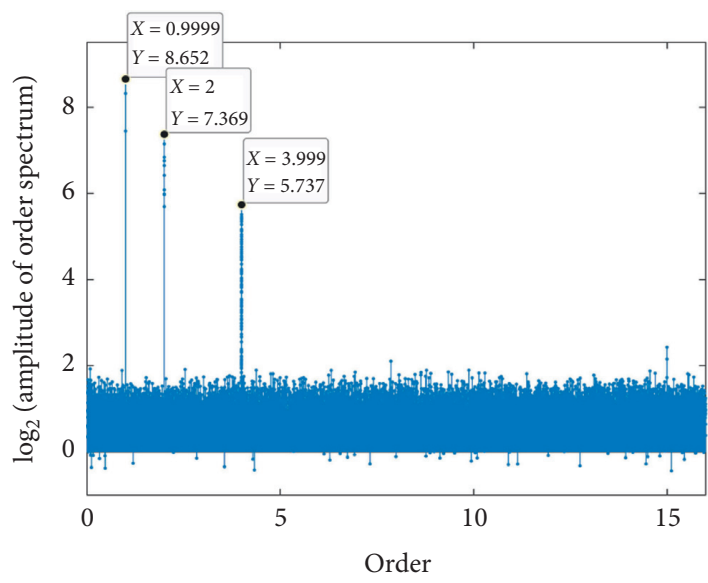

(a)

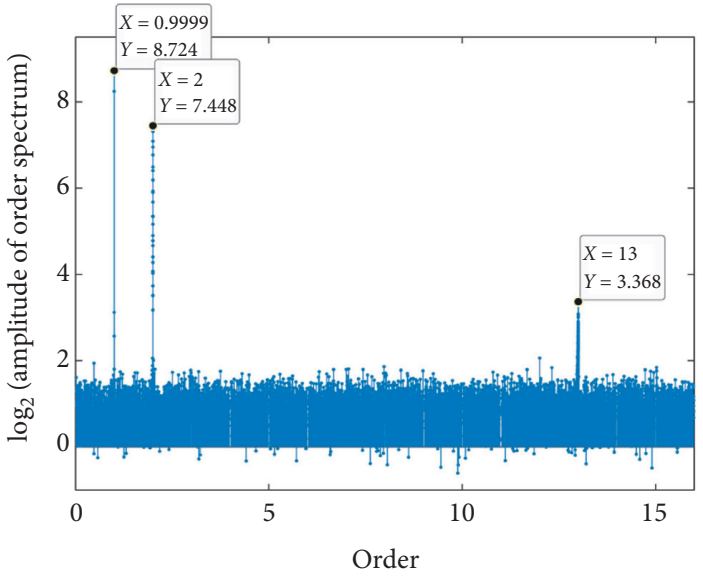

(b)

FIGURE 7: Order spectrums of the simulated blade tip displacements obtained via the CSOA method with random installation angle errors: (a) $\left\{E_{1}=1, E_{2}=2, E_{3}=4\right\}$; (b) $\left\{E_{1}=1, E_{2}=2, E_{3}=13\right\}$.

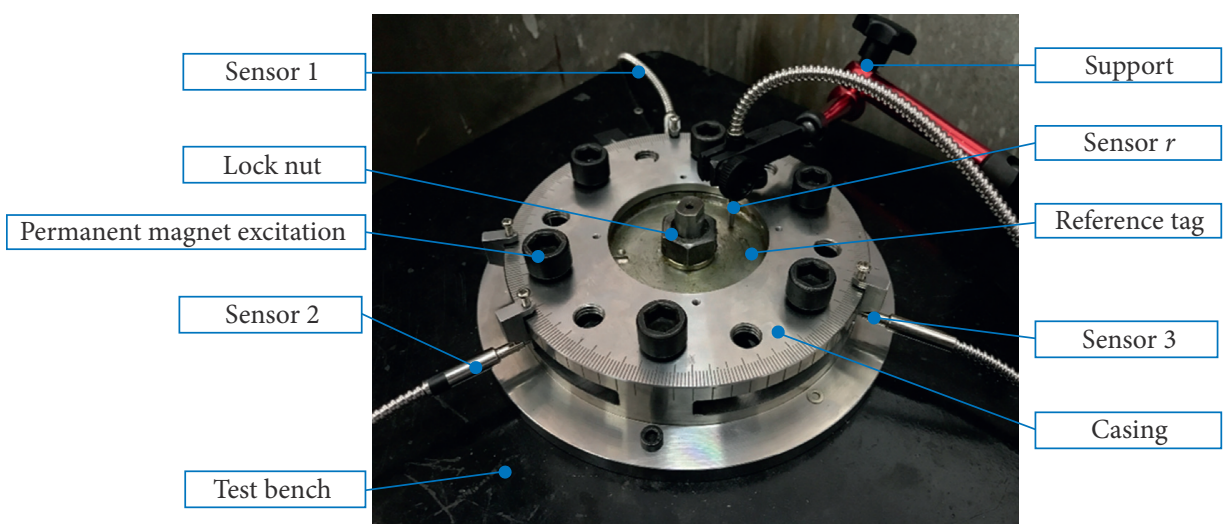

Figure 8: Experimental rig. 
TABle 2: Parameters of the blades.

\begin{tabular}{lr}
\hline Parameters & Values \\
\hline Material & $40 \mathrm{Cr}$ \\
Length & $34 \mathrm{~mm}$ \\
Width & $22 \mathrm{~mm}$ \\
Thickness & $3 \mathrm{~mm}$ \\
Blade tip radius & $69 \mathrm{~mm}$ \\
\hline
\end{tabular}

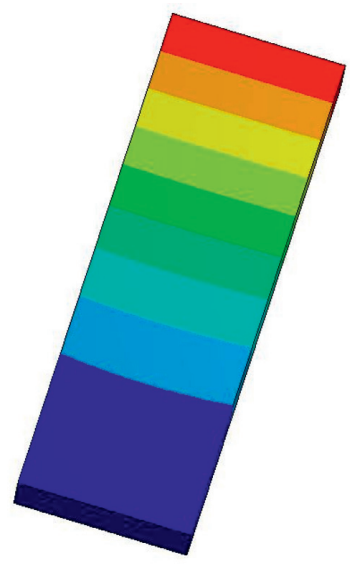

(a)

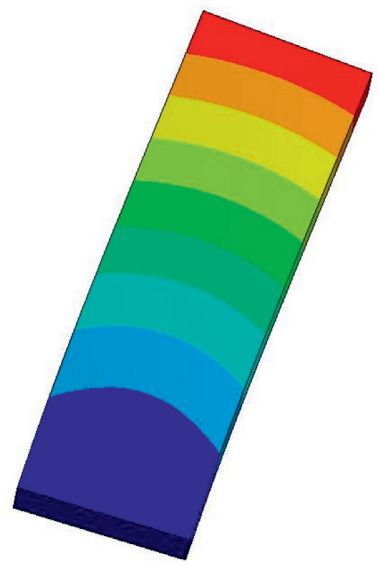

(b)

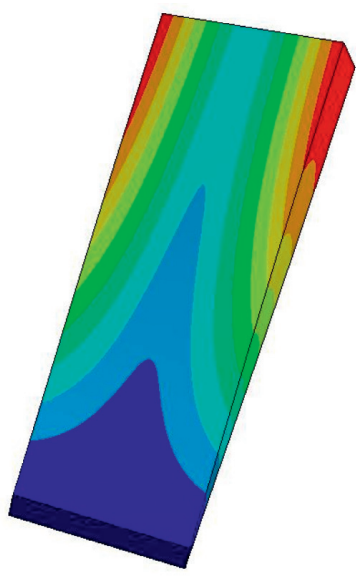

(c)

Figure 9: Modal shapes of the first three vibration modes of the blade model: (a) the first mode; (b) the second mode; (c) the third mode.

were corrected into $15.39^{\circ}, 143.07^{\circ}$, and $270.51^{\circ}$ using the TOAs measured by the sensors at a constant speed. The parameters of the blades are presented in Table 2.

Finite element simulation analysis of the blade was conducted according to the parameters in Table 2. Figure 9 shows that the modal shapes of the first three vibration modes were bending, bending, and torsion, respectively. And Figure 10 shows the Campbell diagram of the blade model. Point A $(4397,2199)$ was selected, where the blade resonated at the first mode and 30 times the rotating frequency. Thus, the rotating speed was varied around point A to obtain blade resonant vibration.

According to the modal analysis result of the blade, the rotating speed was increased continuously from $3850 \mathrm{rpm}$ to $4850 \mathrm{rpm}$. Blade 27 was arbitrarily chosen for analysis. The blade tip displacement varied with the rotating speed and maximized at the resonant speed range (RSR), $4422 \sim 4426 \mathrm{rpm}$ (Figure 11(a)), which was close to the modal analysis result. Figure 11(b) shows that the blade vibrated at 30 times the rotating frequency. The consistency of the modal analysis and experimental result provided a strong evidence for the validity of the CSOA method.

The experimental data of all the blades were further analyzed with the CSOA method and the two methods in [18], as shown in Table 3. $E_{\mathrm{CSOA}}$ represents the vibration order obtained by the CSOA method. $E_{M 1}^{(i)}$ and $E_{M 2}^{(i)}$ are the vibration orders identified, respectively, by the existing two methods with the presupposed central order (PCO) equal to $i$. The RSRs were between $4387 \mathrm{rpm}$ and $4448 \mathrm{rpm}$, which

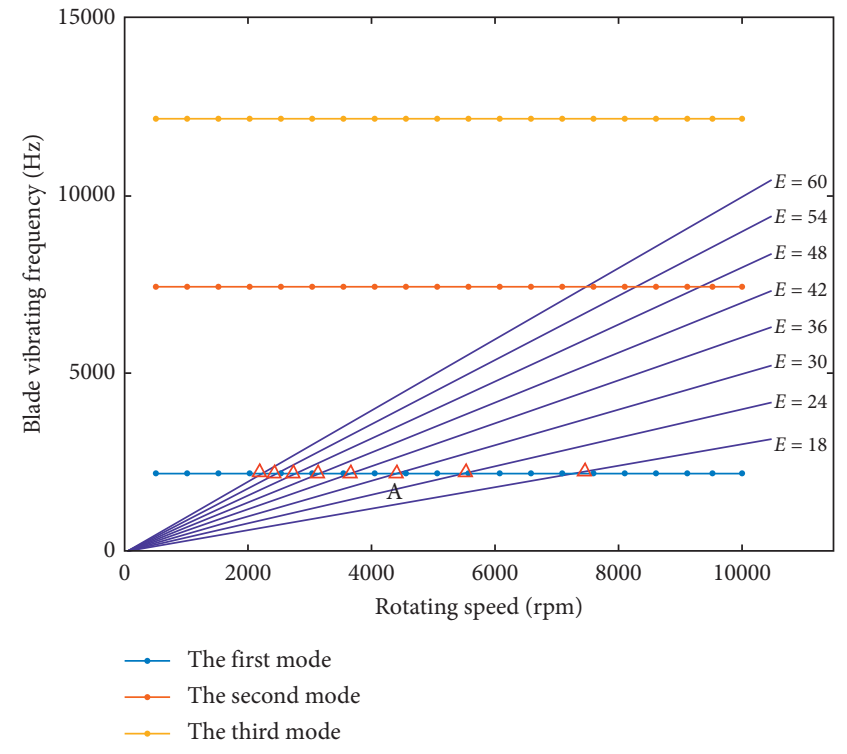

Figure 10: Campbell diagram of blades.

suggested that the blades all vibrated at 30 times of the rotating frequency. $E_{\mathrm{CSOA}}$ varied from 29.97 to 30.06 , which further validated the proposed CSOA method. When the PCO was set as 30 , both existing methods identified the vibration orders precisely. However, the changes in PCO lead to incorrect results, which demonstrated that the success of these methods depended on the PCO. Thus, the 


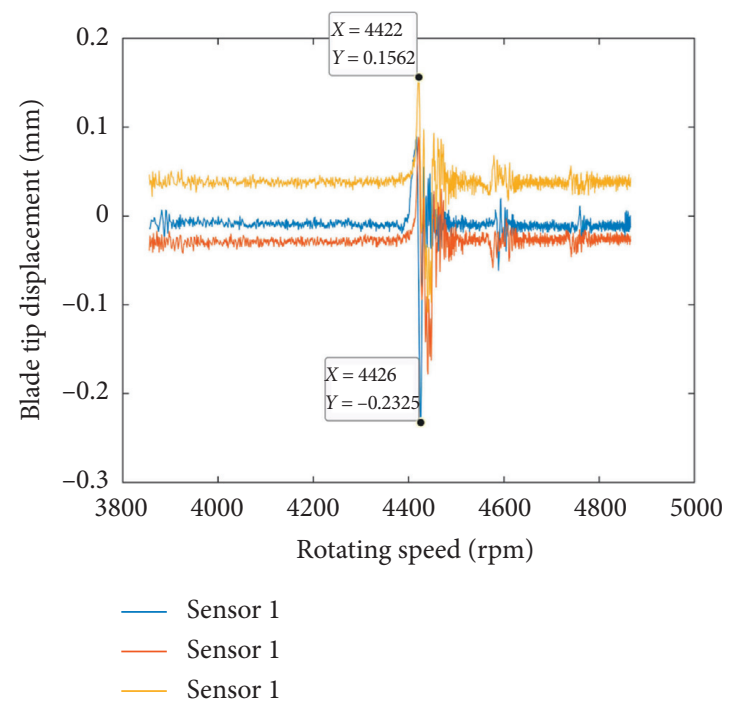

(a)

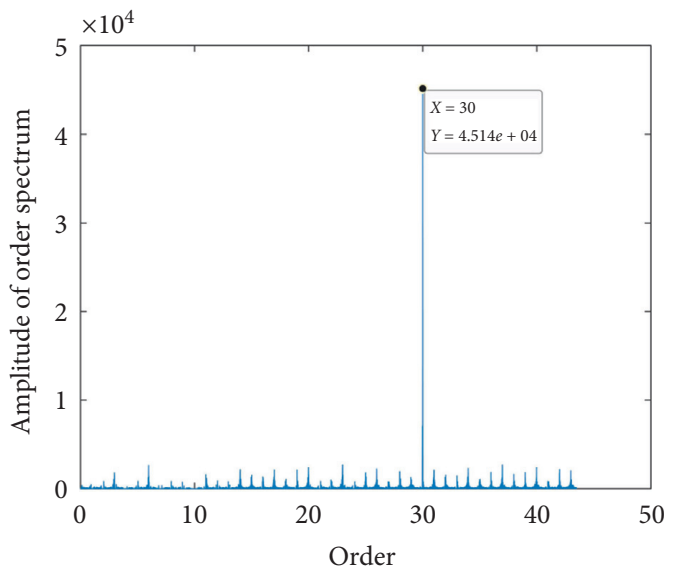

Figure 11: Experimental result of Blade 27: (a) blade tip displacement varied with rotating speed and was sampled by Sensors 1 3; (b) order spectrum of blade vibration obtained by the CSOA method.

TABLE 3: Experimental results of all blades.

\begin{tabular}{|c|c|c|c|c|c|c|}
\hline Blade & $\mathrm{RSP}(\mathrm{rpm})$ & $E_{\mathrm{CSOA}}$ & $E_{M 1}^{(30)}$ & $E_{M 1}^{(18)}$ & $E_{M 2}^{(30)}$ & $E_{M 2}^{(13)}$ \\
\hline 1 & $4405 \sim 4413$ & 30.06 & 30 & 18 & 30 & 14 \\
\hline 2 & $4405 \sim 4422$ & 29.97 & 30 & 18 & 30 & 14 \\
\hline 3 & $4403 \sim 4438$ & 29.99 & 30 & 18 & 30 & 14 \\
\hline 4 & $4431 \sim 4439$ & 30.03 & 30 & 18 & 30 & 14 \\
\hline 5 & $4400 \sim 4408$ & 30.02 & 30 & 18 & 30 & 14 \\
\hline 6 & $4409 \sim 4417$ & 29.99 & 30 & 18 & 30 & 14 \\
\hline 7 & $4410 \sim 4417$ & 30.01 & 30 & 18 & 30 & 14 \\
\hline 8 & $4402 \sim 4426$ & 30.02 & 30 & 18 & 30 & 14 \\
\hline 9 & $4387 \sim 4403$ & 30.04 & 30 & 18 & 30 & 14 \\
\hline 10 & $4403 \sim 4413$ & 30.01 & 30 & 18 & 30 & 14 \\
\hline 11 & $4413 \sim 4414$ & 30 & 30 & 18 & 30 & 14 \\
\hline 12 & $4413 \sim 4413$ & 30.03 & 30 & 18 & 30 & 14 \\
\hline 13 & $4404 \sim 4413$ & 30.01 & 30 & 18 & 30 & 14 \\
\hline 14 & $4411 \sim 4425$ & 30 & 30 & 18 & 30 & 14 \\
\hline 15 & $4403 \sim 4433$ & 30.01 & 30 & 18 & 30 & 14 \\
\hline 16 & $4425 \sim 4441$ & 29.97 & 30 & 18 & 30 & 14 \\
\hline 17 & $4420 \sim 4426$ & 30.02 & 30 & 18 & 30 & 14 \\
\hline 18 & $4441 \sim 4448$ & 30 & 30 & 18 & 30 & 14 \\
\hline 19 & $4409 \sim 4409$ & 30 & 30 & 18 & 30 & 14 \\
\hline 20 & $4408 \sim 4415$ & 30.05 & 30 & 18 & 30 & 14 \\
\hline 21 & $4423 \sim 4424$ & 30.05 & 30 & 18 & 30 & 14 \\
\hline 22 & $4430 \sim 4437$ & 30.02 & 30 & 18 & 30 & 14 \\
\hline 23 & $4416 \sim 4440$ & 29.99 & 30 & 18 & 30 & 14 \\
\hline 24 & $4416 \sim 4426$ & 30 & 30 & 18 & 30 & 14 \\
\hline 25 & $4406 \sim 4433$ & 29.97 & 30 & 18 & 30 & 14 \\
\hline 26 & $4405 \sim 4413$ & 30.02 & 30 & 18 & 30 & 14 \\
\hline 27 & $4422 \sim 4426$ & 30 & 30 & 18 & 30 & 14 \\
\hline 28 & $4424 \sim 4436$ & 29.98 & 30 & 18 & 30 & 14 \\
\hline 29 & $4407 \sim 4439$ & 29.99 & 30 & 18 & 30 & 14 \\
\hline 30 & $4407 \sim 4407$ & 29.99 & 30 & 18 & 30 & 14 \\
\hline 31 & $4408 \sim 4436$ & 30 & 30.05 & 18.05 & 30 & 14 \\
\hline 32 & $4403 \sim 4437$ & 29.97 & 30 & 18 & 30 & 14 \\
\hline
\end{tabular}


CSOA method has an advantage over the existing two methods when the central order of blade vibration is unknown.

\section{Conclusions}

Currently, BTT is considered as a promising technique for online monitoring blade vibrations at a high-rotational speed. However, VRS brings obstacles to extracting blade vibration characteristics from undersampled BTT signals. To solve this problem, this paper proposed a CSOA method for BTT signals measured at VRS, which has good advantages over the existing methods. A CSOA mathematical model was built. Simulations and experiments were performed to demonstrate the advantages and validity of the CSOA method. The main results are summarized as follows:

(1) The order spectrum of undersampled BTT vibration signals measured at VRS can be accurately obtained through the proposed CSOA method.

(2) No prior information of the central order and order bandwidth of BTT signals is needed for analysis via the CSOA method.

(3) The CSOA method is effective whether the BTT vibration signals are within or exceeding the order bandwidth limitation of the existing methods in [18].

(4) The CSOA method allows considerable installation angle errors, which is more applicable to the actual situation.

\section{Data Availability}

The data used to support this research are available from the corresponding author upon request.

\section{Conflicts of Interest}

The authors declare that they have no conflicts of interest.

\section{Acknowledgments}

This research was supported by the National Basic Research Program of China (Grant no. 2015CB057404) and the Natural Science Foundation of Hunan Province, China (Grant no. 2019JJ50721).

\section{References}

[1] H. Guo, F. Duan, and J. Zhang, "Blade resonance parameter identification based on tip-timing method without the onceper revolution sensor," Mechanical Systems and Signal Processing, vol. 66-67, pp. 625-639, 2016.

[2] J.-w. Zhang, L.-b. Zhang, and L.-x. Duan, "A blade defect diagnosis method by fusing blade tip timing and tip clearance information," Sensors, vol. 18, no. 7, p. 2166, 2018.

[3] G. Dimitriadis, I. B. Carrington, J. R. Wright, and J. E. Cooper, "Blade-tip timing measurement of synchronous vibrations of rotating bladed assemblies," Mechanical Systems and Signal Processing, vol. 16, no. 4, pp. 599-622, 2002.

[4] D. Knappett and J. Garcia, "Blade tip timing and strain gauge correlation on compressor blades," Proceedings of the
Institution of Mechanical Engineers, Part G: Journal of Aerospace Engineering, vol. 222, no. 4, pp. 497-506, 2008.

[5] G. Rigosi, G. Battiato, and T. M. Berruti, "Synchronous vibration parameters identification by tip timing measurements," Mechanics Research Communications, vol. 79, pp. 7-14, 2017.

[6] T. M. Pickering, "Methods for validation of a turbomachinery rotor blade tip timing system," Virginia Tech, 2014.

[7] F. Duan, J. Zhang, J. Jiang, H. Guo, and D. Ye, "Method to improve the blade tip-timing accuracy of fiber bundle sensor under varying tip clearance," Optical Engineering, vol. 55, no. 1, Article ID 014106, 2016.

[8] J. Zhang, F. Duan, G. Niu, J. Jiang, and J. Li, "A blade tip timing method based on a microwave sensor," Sensors, vol. 17, no. 5, p. 1097, 2017.

[9] Z. Hu, J. Lin, Z.-S. Chen, Y.-M. Yang, and X.-J. Li, “A nonuniformly under-sampled blade tip-timing signal reconstruction method for blade vibration monitoring," Sensors, vol. 15, no. 2, pp. 2419-2437, 2015.

[10] F. Figaschewsky, A. Kuhhorn, and T. Giersch, "A higher order linear least square fit for the assessment of integral and NonIntegral vibrations with blade tip timing," in Proceedings of the 17th International Symposium on Transport Phenomena and Dynamics of Rotating Machinery, Maui, HI, USA, December 2017.

[11] C. E. Shannon, "Communication in the presence of noise," Proceedings of the IRE, vol. 37, no. 1, pp. 10-21, 1949.

[12] M. Witos and M. Wisnioch, "Compressor blade health monitoring with use of tip timing and modal analysis method," Pamm, vol. 9, no. 1, pp. 209-212, 2010.

[13] P. Beauseroy and R. Lengellé, "Nonintrusive turbomachine blade vibration measurement system," Mechanical Systems and Signal Processing, vol. 21, no. 4, pp. 1717-1738, 2007.

[14] B. Salhi, J. Lardiès, M. Berthillier, P. Voinis, and C. Bodel, "Modal parameter identification of mistuned bladed disks using tip timing data," Journal of Sound and Vibration, vol. 314, no. 3-5, pp. 885-906, 2008.

[15] J. Lin, Z. Hu, Z.-S. Chen, Y.-M. Yang, and H.-L. Xu, "Sparse reconstruction of blade tip-timing signals for multi-mode blade vibration monitoring," Mechanical Systems and Signal Processing, vol. 81, pp. 250-258, 2016.

[16] M. Pan, Y. Yang, F. Guan, H. Hu, and H. Xu, "Sparse representation based frequency detection and uncertainty reduction in blade tip timing measurement for multi-mode blade vibration monitoring," Sensors, vol. 17, no. 8, 2017.

[17] C. Zhan, Z. Chen, Z. Hu, Y. Yang, and H. Hu, "Nonlinear time transformation-based vibration signal reconstruction for blade tip-timing under rotation speed fluctuations," pp. 1-5, 2017.

[18] Z. Chen, J. Liu, C. Zhan, J. He, and W. Wang, "Reconstructed order analysis-based vibration monitoring under variable rotation speed by using multiple blade tip-timing sensors," Sensors, vol. 18, no. 10, p. 3235, 2018.

[19] D. L. Donoho, "Compressed sensing," Ieee Transactions On Information Theory, vol. 52, no. 4, pp. 1289-1306, 2006.

[20] E. J. Candès and J. Emmanuel, "The restricted isometry property and its implications for compressed sensing," Comptes Rendus Mathematique, vol. 346, no. 9-10, pp. 589-592, 2008.

[21] M. Mishali and Y. C. Eldar, "Blind multiband signal reconstruction: compressed sensing for analog signals," Ieee Transactions On Signal Processing, vol. 57, no. 3, pp. 993-1009, 2009. 
[22] R. G. Baraniuk, V. Cevher, M. F. Duarte, and C. Hegde, "Model-based compressive sensing," IEEE Transactions on Information Theory, vol. 56, no. 4, pp. 1982-2001, 2010.

[23] D. L. Donoho and M. Elad, "Optimally sparse representation in general (nonorthogonal) dictionaries via 1 minimization," Proceedings of the National Academy of Sciences, vol. 100, no. 5, pp. 2197-2202, 2003.

[24] D. L. Donoho and X. Huo, "Uncertainty principles and ideal atomic decomposition," IEEE Transactions on Information Theory, vol. 47, no. 7, pp. 2845-2862, 2001.

[25] M. Elad, "Sparse and redundant representations: from theory to applications in signal and image processing," pp. 10941097, 2010.

[26] J. A. Tropp, "Greed is good: algorithmic results for sparse approximation," Ieee Transactions On Information Theory, vol. 50, no. 10, pp. 2231-2242, 2004.

[27] J. A. Tropp, I. S. Dhillon, R. W. Heath, and T. Strohmer, "Designing structured tight frames via an alternating projection method," Ieee Transactions On Information Theory, vol. 51, no. 1, pp. 188-209, 2005.

[28] J. A. Tropp and A. C. Gilbert, "Signal recovery from random measurements via orthogonal matching pursuit," Ieee Transactions On Information Theory, vol. 53, no. 12, pp. 4655-4666, 2007.

[29] D. Needell and R. Vershynin, "Signal recovery from incomplete and inaccurate measurements via regularized orthogonal matching pursuit," IEEE Journal of Selected Topics in Signal Processing, vol. 4, no. 2, pp. 310-316, 2010.

[30] S. S. Chen, D. L. Donoho, and M. A. Saunders, "Atomic decomposition by basis pursuit," Siam Review, vol. 43, no. 1, pp. 129-159, 2001.

[31] D. L. Donoho and M. Elad, "On the stability of the basis pursuit in the presence of noise," Signal Processing, vol. 86, no. 3, pp. 511-532, 2006.

[32] A. M. Bruckstein, M. Elad, and M. Zibulevsky, "On the uniqueness of nonnegative sparse solutions to underdetermined systems of equations," Ieee Transactions On Information Theory, vol. 54, no. 11, pp. 4813-4820, 2008.

[33] J. G. Proakis and D. G. Manolakis, "Digital signal processing: principles, algorithms, and applications,” pp. 392-394, 1996. 